\title{
A UTILIZAÇÃO DO NINTENDO WII® COMO FERRAMENTA TERAPÊUTICA EM PROGRAMAS DE REABILITAÇÃO TRAUMATO-ORTOPÉDICA
}

-uma revisão de literatura•

\author{
Rafael Vinícius Santos Cruz*, Matheus Silva d'Alencar**, Marcos Rodrigo Trindade Pinheiro \\ Menuchi*** \\ Autor correspondente: Rafael Vinícius Santos Cruz - E-mail: rafa.fisio7@hotmail.com \\ * Mestrando em Desenvolvimento e Meio Ambiente pela Universidade Estadual de Santa Cruz (UESC); Fisioterapeuta na \\ Unidade Cardiointensiva do Hospital Calixto Midlej Filho, Santa Casa de Misericórdia de Itabuna-BA \\ ** Fisioterapeuta no Hospital Geral Luiz Viana Filho, Ilhéus-BA; Mestre em Tecnologias em Saúde pela Escola Bahiana de \\ Medicina e Saúde Pública. \\ *** Mestre em Ciência da Motricidade pela Universidade Estadual Paulista (Rio Claro); Professor do Departamento de \\ Ciências da Saúde da Universidade Estadual de Santa Cruz (UESC).
}

\section{Resumo}

Objetivo: investigar a utilização do Nintendo Wii $®$ como ferramenta central ou adjuvante em programas de reabilitação de pacientes com problemas traumato-ortopédicos. Metodologia: $O$ estudo consiste em uma revisão de literatura. Foram pesquisados artigos nos idiomas inglês e/ou português, disponíveis na íntegra, cadastrados nas bases eletrônicas de pesquisa Pubmed Central, Scielo, Lilacs, Bireme, Cochrane, Medline e PEDro, publicados entre 2006 e 2014. Resultados: sete artigos corresponderam aos critérios de inclusão estabelecidos, sendo seis em idioma inglês, e um, em português. Todos eles foram apresentados como randomizados, quatro destes avaliaram indivíduos com problemas em membro inferior, com atenção especial à articulação do joelho, sendo dois voltados para o tratamento de lombalgia e um para disfunção do membro superior. Exercícios de fortalecimento foram comuns a todos os estudos, sendo também comum o emprego de exercícios para melhora do equilíbrio corporal e coordenação. Quanto aos jogos utilizados no Nintendo Wii $\circledast$, houve uma diversificação, com o emprego de games direcionados ao equilíbrio, à propriocepção, ao fortalecimento, ao alongamento e ao condicionamento aeróbico. A duração do programa variou entre 4 e 12 semanas, com 2 a 4 sessões semanais de exercício ou wiiterapia. Conclusão: a utilização de protocolos de intervenção com o uso do Nintendo Wii $®$ para distúrbios traumato-ortopédicos mostrou resultados positivos nas disfunções de membros superiores, membros inferiores e coluna.

Palavras-chave: Ortopedia; Traumatologia; Terapia de Exposição à Realidade Virtual. 


\title{
THE NINTENDO WII ${ }^{\circledR}$ USE AS THERAPEUTIC TOOL IN REHABILITATION PROGRAMS TRAUMATIC AND ORTHOPEDIC
}

\author{
- a literature review•
}

\begin{abstract}
Objective: investigate the use of the Nintendo Wii as a central tool or adjuvant in patient rehabilitation programs with trauma-orthopedic problems. Methodology: The study consists of a literature review. Articles were searched in English and / or Portuguese, available in full, registered in the electronic databases to search PubMed Central, SciELO, Lilacs, Bireme, Cochrane, Medline and PEDro, published between 2006 and 2014. Results: 7 articles that correspond to criteria inclusion, six in english and one in português. All articles were presented as randomized, four of these evaluated individuals with lower limb problems, with special attention to the knee joint, two focused the treatment of low back pain and one for the upper limb dysfunction. Strengthening exercises were common to all studies and is also common the use of exercises to improve body balance and coordination. As for games used in the Nintendo Wii, there was a diversification, with the games of employment targeted to balance, to proprioception, strengthening, stretching and aerobic to the character. The program duration ranged from 4 to 12 weeks, with 2-4 weekly sessions of exercise or wiitherapy. Conclusion: The use of Nintendo $\mathrm{Wii} \circledast$ protocols for traumatic and orthopedic disorders showed positive results in upper limbs, lower limbs and column dysfunction.
\end{abstract}

Keywords: Orthopedics; Traumatology; Virtual Reality Exposure Therapy.

\section{INTRODUÇÃO}

Os exergames, consoles que buscam quebrar o estigma social dos vídeogames como fomentadores de um estilo de vida sedentário, têm atuado na contramão desta antiga tendência, já que suas novas plataformas computacionais e softwares estão procurando incrementar o movimento corporal como parte integrante e fundamental da jogabilidade de seus lançamentos. Esse fenômeno teve o seu grande estopim em 2006, com o lançamento do Nintendo Wiiß.(1)

O sistema do Nintendo $\mathrm{Wii} \circledast$ é pautado em sensores infravermelhos e acelerômetros internos para captação dos movimentos, com a utilização de um sistema de feedback instantâneo sensório- motor. ${ }^{(2)}$ Esse sistema motiva o usuário através de uma interface intuitiva e sistema de pontuações, fazendo com que sua prática seja divertida e eficaz de forma simultânea. (3) Além disso, constitui em um poderoso aliado na continuidade de tratamentos fisioterapêuticos em domicílio. ${ }^{(4)}$

A realidade virtual da qual os exergames se valem envolvem três aspectos principais: imersão, interação e envolvimento. Tais características são de extremo interesse na elaboração de um programa terapêutico que busque manter a adesão do paciente durante o tempo necessário através da motivação e do seu caráter desafiador, garantindo o efeito almejado pela reabilitação. $(5,6)$ 
As respostas fisiológicas e a utilização do Nintendo $\mathrm{Wii} \circledast$ em programas terapêuticos já foram estudadas nos campos da reabilitação neurológica, ${ }^{(7,8)}$ da reabilitação cardiovascular ${ }^{(9,10)}$ e no combate à obesidade, ${ }^{(11,12)}$ bem como alguns relatos de efeitos adversos resultantes da sua utilização, como mialgias agudas em extremidades superiores, ${ }^{(13)}$ tendinites ${ }^{(14)}$ e desvios posturais. ${ }^{(15)}$ Seu uso para melhora do aspecto da mobilidade funcional tem sido discutido em estudos com pacientes pós -Acidente Vascular Encefálico ${ }^{(16)}$ e pós-doença de Parkinson. ${ }^{(17)}$ Pouca informação, entretanto, se tem acerca do seu emprego em reabilitações traumato -ortopédicas e a eficácia de sua aplicação.

Diante deste cenário, o presente estudo tem como objetivo investigar a utilização do Nintendo Wii $®$ como ferramenta central ou adjuvante em programas de reabilitação de pacientes com problemas traumato-ortopédicos.

\section{METODOLOGIA}

A pesquisa consistiu em uma revisão de literatura acerca de estudos que demonstraram a utilização do Nintendo Wiiß como parte integrante de um programa de reabilitação em pacientes com problemas traumato-ortopédicos. Foram pesquisados artigos nos idiomas inglês e/ou português, disponíveis na íntegra, cadastrados nas bases eletrônicas de pesquisa Pubmed Central, Scielo, Lilacs, Bireme, Cochrane, Medline e PEDro.

Utilizaram-se, como termos de busca, as palavras-chave e seus respectivos correspondentes linguísticos: Nintendo Wii, wiiterapia (wiitherapy), reabilitação com wii (wii rehabilitation) de forma isolada ou combinada com as seguintes palavras lesão osteomuscular (osteomuscular injury), lesão traumática (traumatic injury), doença osteomuscular (osteomuscular disease), ortopedia (orthopedia), fortalecimento (strenght). Foi determinado como limites na busca os artigos publicados a partir do ano de 2006 (ano de lançamento do Nintendo Wiiß) até dezembro de 2014, na qual a fase de pesquisa foi concluída.
Foram excluidos os artigos que tratavam como tema central condições patológicas diferentes das traumato-ortopédicas, a saber: condições neurológicas, cardiovasculares, distúrbios vestibulares, renais, assim como artigos que avaliaram indivíduos saudáveis, em qualquer idade, ou que utilizaram o

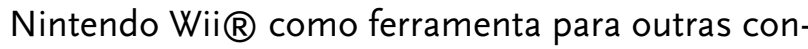
dições, como a redução de peso ou incremento no nível de atividade física. Excluiu-se também estudos que utilizaram o Nintendo $\mathrm{Wii} \circledast$ ou seus elementos de forma adaptada a outros softwares ou sistemas computacionais, visto que o intuito desta revisão foi investigar se o console, no seu modelo comercial e sem modificações de software ou har$d w a r e$, pode ser útil em programas de reabilitação traumato-ortopédicas.

Durante a pesquisa, os artigos foram selecionados baseados em seus títulos e leitura dos resumos. Após esta etapa, os estudos aprovados na primeira fase passaram por uma minuciosa leitura para averiguação dos critérios de inclusão, sendo excluídos aqueles que não correspondiam aos termos pré-estabelecidos. Os artigos selecionados após o crivo final foram organizados na forma de tabela com a delimitação de seus aspectos principais, a saber: autor, país, amostra, grupo controle, intervenções, duração do programa, ferramentas de avaliação e conclusões alcançadas.

\section{RESULTADOS}

Foram encontrados 10 artigos, sendo que três foram excluídos: o primeiro, por utilizar o Nintendo Wiiß unicamente como ferramenta de avaliação, o segundo, por ter, na sua amostra, indivíduos com problemas ortopédicos porém não como eixo central do estudo, e o terceiro, por utilizar o Nintendo Wii $®$ em uma forma modificada associada a um software computacional externo. Foram selecionados sete artigos que correspondiam aos critérios de inclusão estabelecidos, sendo seis em idioma inglês e um, em português. Quanto à nacionalidade, o Canadá e a Coréia do Sul apresentaram dois estudos cada, seguido por Brasil, Estados 
Unidos e Turquia, com um estudo cada. Todos eles foram apresentados como randomizados, sendo que quatro destes avaliaram indivíduos com problemas em membro inferior, com atenção especial à articulação do joelho, outros dois voltaram para o tratamento de lombalgia e um direcionou para disfunção do membro superior (Tabela 1).

Exercícios de fortalecimento foram comuns a todos os estudos, sendo também comum o emprego de exercícios para melhora do equilíbrio corporal e coordenação. Quanto aos jogos utilizados no Nintendo $\mathrm{Wii} \circledast$, houve uma diversificação, com o emprego de games direcionados ao equilíbrio, à propriocepção, ao fortalecimento, ao alongamento e ao condicionamento aeróbico. A duração do programa variou de quatro a 12 semanas, com protocolos que incluíram entre duas e quatro sessões semanais de exercício ou wiiterapia.

Tabela 1 - Sumário dos estudos que empregaram o Nintendo Wii para Reabilitação Traumato-ortopédica

(continua)

\begin{tabular}{|c|c|c|c|c|c|c|c|c|}
\hline AUTOR/ANO & PAís & $\begin{array}{l}\text { TIPO DE } \\
\text { ESTUDO }\end{array}$ & AMOSTRA & $\begin{array}{c}\text { GRUPO } \\
\text { CONTROLE }\end{array}$ & INTERVENÇÃO & $\begin{array}{l}\text { DURAÇÃO DO } \\
\text { PROGRAMA }\end{array}$ & $\begin{array}{l}\text { INSTRUMENTOS } \\
\text { DE AVALIAÇÃO }\end{array}$ & CONCLUSÕES \\
\hline $\begin{array}{l}\text { Kim, Min, } \\
\text { Kim, Lee } \\
(2014)\end{array}$ & $\begin{array}{l}\text { Coréia do } \\
\text { Sul }\end{array}$ & $\begin{array}{l}\text { Rando- } \\
\text { mizado, } \\
\text { controlado }\end{array}$ & $\begin{array}{l}30 \text { mulheres } \\
\text { de meia } \\
\text { idade com } \\
\text { diagnóstico } \\
\text { clínico de } \\
\text { dor lombar }\end{array}$ & $\begin{array}{l}\text { Exercícios } \\
\text { de estabi- } \\
\text { lização do } \\
\text { tronco e } \\
\text { fisioterapia } \\
\text { convencio- } \\
\text { nal ( } n=15)\end{array}$ & $\begin{array}{l}\text { Programa de } \\
\text { loga com a } \\
\text { utilização do } \\
\text { Wii Fit com } \\
\text { atividades } \\
\text { como respira- } \\
\text { ção profunda, } \\
\text { posição Meia } \\
\text { Lua, Guerreiro, } \\
\text { Árvore, Tórax } \\
\text { ao joelho, Ca- } \\
\text { deira, Palmeira } \\
(n=15)\end{array}$ & $\begin{array}{l}12 \text { sessões ao } \\
\text { longo de qua- } \\
\text { tro semanas } \\
\text { com duração } \\
\text { média de } 30 \\
\text { minutos/sessão } \\
\text { para Grupo } \\
\text { Intervenção } \\
\text { e } 60 \text { minutos } \\
\text { para grupo } \\
\text { controle }\end{array}$ & $\begin{array}{l}\text { Escala Visual } \\
\text { Analógica, } \\
\text { Algometria } \\
\text { de pressão, } \\
\text { Oswestry } \\
\text { low-back pain } \\
\text { disability } \\
\text { index (ODI), } \\
\text { Roland Morris } \\
\text { Disability Index } \\
\text { (RDQ), Fear } \\
\text { Avoidance Be- } \\
\text { liefs Question- } \\
\text { naire (FBQ) }\end{array}$ & $\begin{array}{l}\text { Ambos os } \\
\text { programas } \\
\text { demonstraram } \\
\text { ser benéficos } \\
\text { para todas } \\
\text { as variáveis } \\
\text { analisadas }\end{array}$ \\
\hline $\begin{array}{l}\text { Wibelinger, } \\
\text { Batista, } \\
\text { Kayser, } \\
\text { Pasqualotti } \\
\text { (2013) }\end{array}$ & Brasil & $\begin{array}{l}\text { Rando- } \\
\text { mizado, } \\
\text { controlado }\end{array}$ & $\begin{array}{l}71 \text { mulheres } \\
\text { idosas com } \\
\text { diagnóstico } \\
\text { de osteoartri- } \\
\text { te de joelho } \\
\text { e apresen- } \\
\text { tando dor } \\
\text { articular }\end{array}$ & $\begin{array}{l}\text { Técnicas de } \\
\text { respiração, } \\
\text { fortaleci- } \\
\text { mento de } \\
\text { membros } \\
\text { inferiores, } \\
\text { desloca- } \\
\text { mento } \\
\text { multidirecio- } \\
\text { nal, treino } \\
\text { de marcha, } \\
\text { step ( } n=33 \text { ) }\end{array}$ & $\begin{array}{l}\text { loga, exercícios } \\
\text { de equilíbrio } \\
\text { (Penguim Slide, } \\
\text { Ski Slalom, Soc- } \\
\text { cer Heading), } \\
\text { Tightrope Walk } \\
\text { e exercícios de } \\
\text { deslocamento } \\
\text { multidirecional, } \\
\text { como o Table } \\
\text { Tilt ( } \mathrm{n}=38 \text { ) }\end{array}$ & $\begin{array}{l}\text { Duas vezes por } \\
\text { semana, Grupo } \\
\text { Controle com } \\
50 \text { minutos/ } \\
\text { sessão e Grupo } \\
\text { Intervenção } \\
\text { com } 30 \\
\text { minutos, por } 3 \\
\text { meses }\end{array}$ & $\begin{array}{l}\text { Questionário } \\
\text { acerca da } \\
\text { dor, Western } \\
\text { Ontario and } \\
\text { McMaster } \\
\text { Osteoarthritis } \\
\text { Index (WO- } \\
\text { MAC), Escala } \\
\text { de Equilíbrio } \\
\text { de Berg }\end{array}$ & $\begin{array}{l}\text { Ambos os } \\
\text { grupos foram } \\
\text { efetivos no } \\
\text { tratamento, } \\
\text { porém o grupo } \\
\text { que utilizou } \\
\text { o Wii obteve } \\
\text { superioridade } \\
\text { nos itens equi- } \\
\text { líbrio e rigidez } \\
\text { articular }\end{array}$ \\
\hline $\begin{array}{l}\text { Sims, } \\
\text { Cosby, } \\
\text { Saliba, } \\
\text { Hertel } \\
\text { (2013) }\end{array}$ & EUA & $\begin{array}{l}\text { Randomiza- } \\
\text { do, contro- } \\
\text { lado, cego } \\
\text { simples }\end{array}$ & $\begin{array}{l}25 \text { indivíduos } \\
\text { fisicamente } \\
\text { ativos com } \\
\text { história de } \\
\text { lesão em } \\
\text { membro } \\
\text { inferior ou } \\
\text { cirurgia, } \\
\text { incluindo } \\
\text { ligamento, } \\
\text { cápsula ou } \\
\text { dano na } \\
\text { cartilagem } \\
\text { do joelho ou } \\
\text { tornozelo }\end{array}$ & $\begin{array}{l}\text { A um grupo } \\
\text { foi indicado } \\
\text { apenas a } \\
\text { continuida- } \\
\text { de de suas } \\
\text { atividades } \\
\text { cotidianas } \\
\text { (n=9). } \\
\text { Outro grupo } \\
\text { participou } \\
\text { de um } \\
\text { programa } \\
\text { tradicional } \\
\text { de exerci- } \\
\text { cios para } \\
\text { equilibrio } \\
\text { (n=10) }\end{array}$ & $\begin{array}{l}\text { Exercícios de } \\
\text { ioga, fortaleci- } \\
\text { mento e aeróbi- } \\
\text { co utilizando a } \\
\text { balance board } \\
\text { no Wii Fit. Equi- } \\
\text { líbrio (Soccer } \\
\text { Heading, Table } \\
\text { Tilt, Penguim } \\
\text { Slide, Ski Sla- } \\
\text { lom, Balance } \\
\text { Bubble); Força } \\
\text { (Lunge, Single } \\
\text { Leg, Extension, } \\
\text { Sideway Leg } \\
\text { Lift); Aeróbico } \\
\text { (Hula Hoop, Su- } \\
\text { per Hula Hoop, } \\
\text { Basic Step); } \\
\text { loga (Half } \\
\text { Moon, Tree, } \\
\text { Chair) (n=9) }\end{array}$ & $\begin{array}{l}\text { Três vezes } \\
\text { por semana } \\
\text { durante quatro } \\
\text { semanas, } \\
\text { totalizando } 12 \\
\text { sessões }\end{array}$ & $\begin{array}{l}\text { Star Excursion } \\
\text { Balance Test } \\
\text { (SEBT), Time } \\
\text { to Boundary } \\
\text { (TTB), Lower } \\
\text { Extremity Func- } \\
\text { tional Scale } \\
\text { (LEES) }\end{array}$ & $\begin{array}{l}\text { A reabilitação } \\
\text { tradicional } \\
\text { ou com o } \\
\text { emprego do } \\
\text { Nintendo Wii } \\
\text { obtiveram } \\
\text { sucesso em } \\
\text { otimizar o } \\
\text { controle pos- } \\
\text { tural estático } \\
\text { dos indivíduos } \\
\text { analisados }\end{array}$ \\
\hline
\end{tabular}


Tabela 1 - Sumário dos estudos que empregaram o Nintendo Wii para Reabilitação Traumato-ortopédica

(continuação)

\begin{tabular}{|c|c|c|c|c|c|c|c|c|}
\hline AUTOR/ANO & País & $\begin{array}{l}\text { TIPO DE } \\
\text { ESTUDO }\end{array}$ & AMOSTRA & $\begin{array}{c}\text { GRUPO } \\
\text { CONTROLE }\end{array}$ & INTERVENÇÃO & $\begin{array}{l}\text { DURAÇÃO DO } \\
\text { PROGRAMA }\end{array}$ & $\begin{array}{l}\text { INSTRUMENTOS } \\
\text { DE AVALIAÇÃO }\end{array}$ & CONCLUSÕES \\
\hline $\begin{array}{l}\text { Fung, Ho, } \\
\text { Shaffer, } \\
\text { Chung, } \\
\text { Gomez } \\
\text { (2012) }\end{array}$ & Canadá & $\begin{array}{l}\text { Rando- } \\
\text { mizado, } \\
\text { controlado, } \\
\text { preliminar }\end{array}$ & $\begin{array}{l}50 \text { indivíduos } \\
\text { adultos com } \\
\text { história de } \\
\text { artroplastia } \\
\text { total do } \\
\text { joelho }\end{array}$ & $\begin{array}{l}\text { Exercícios } \\
\text { de fortale- } \\
\text { cimento de } \\
\text { membros } \\
\text { inferiores, } \\
\text { equilíbrio, } \\
\text { mudança na } \\
\text { descarga } \\
\text { de peso e } \\
\text { alongamen- } \\
\text { to ( } n=23)\end{array}$ & $\begin{array}{l}\text { Os jogos Deep } \\
\text { Breathing e Ski } \\
\text { Slalom foram } \\
\text { utilizados com } \\
\text { todos. De } \\
\text { acordo com o } \\
\text { progresso in- } \\
\text { dividual, foram } \\
\text { incorporados } \\
\text { outros jogos, } \\
\text { como os de } \\
\text { alterações } \\
\text { latero-laterais } \\
\text { de descarga de } \\
\text { peso (Tigh- } \\
\text { trope Walk, } \\
\text { Penguin Slide); } \\
\text { Alterações } \\
\text { multidirecionais } \\
\text { de descarga } \\
\text { de peso (Table } \\
\text { Tilt, Hula Hoop, } \\
\text { Balance Bub- } \\
\text { ble); equilíbrio } \\
\text { estático e } \\
\text { dinâmico (Half } \\
\text { Moon, Torso } \\
\text { Twist). (n=27) }\end{array}$ & $\begin{array}{l}\text { Sessão com } \\
\text { duração de } \\
15 \text { minutos } \\
\text { para ambos } \\
\text { os grupos, } \\
\text { sendo esta um } \\
\text { complemento } \\
\text { a uma sessão } \\
\text { de fisioterapia } \\
\text { convencional } \\
\text { com duração } \\
\text { de } 60 \text { minutos. } \\
\text { A duração total } \\
\text { do tratamento } \\
\text { não foi prede- } \\
\text { finida por ser } \\
\text { esta também } \\
\text { uma variável } \\
\text { de interesse } \\
\text { na análise de } \\
\text { resultados } \\
\text { (tempo até a } \\
\text { alta do trata- } \\
\text { mento) }\end{array}$ & $\begin{array}{l}\text { Amplitude de } \\
\text { movimento } \\
\text { do joelho, } \\
\text { 2-minute walk } \\
\text { test (2MWT), } \\
\text { Numeric Pain } \\
\text { Rating Scale } \\
\text { (NPRS), Lower } \\
\text { Extremity } \\
\text { Functional } \\
\text { Scale (LEFS), } \\
\text { Activity-spe- } \\
\text { cific Balance } \\
\text { Confidence } \\
\text { Scale (ABCS), } \\
\text { duração da } \\
\text { reabilitação } \\
\text { até a alta. }\end{array}$ & $\begin{array}{l}\text { Não houveram } \\
\text { diferenças } \\
\text { significativas } \\
\text { entre os dois } \\
\text { grupos. Su- } \\
\text { gere-se que o } \\
\text { Nintendo Wii } \\
\text { tem potencial } \\
\text { para exercer } \\
\text { funcão } \\
\text { adjuvante em } \\
\text { programas de } \\
\text { reabilitação } \\
\text { para artro- } \\
\text { plastia total } \\
\text { do joelho. }\end{array}$ \\
\hline $\begin{array}{l}\text { Park, Lee, } \\
\text { Ko (2013) }\end{array}$ & $\begin{array}{l}\text { Coréia do } \\
\text { Sul }\end{array}$ & $\begin{array}{l}\text { Rando- } \\
\text { mizado, } \\
\text { controlado }\end{array}$ & $\begin{array}{l}24 \text { trabalha- } \\
\text { dores de uma } \\
\text { fábrica de } \\
\text { pneus, com } \\
\text { diagnóstico } \\
\text { clínico de } \\
\text { lombalgia }\end{array}$ & $\begin{array}{l}\text { O grupo } \\
\text { controle } \\
\text { (GC) foi } \\
\text { compos- } \\
\text { to por } 8 \\
\text { indivíduos } \\
\text { submetidos } \\
\text { a recursos } \\
\text { eletrotérmi- } \\
\text { cos. Outro } \\
\text { grupo teve } \\
\text { em seu } \\
\text { programa } \\
\text { além dos } \\
\text { recursos an- } \\
\text { teriormente } \\
\text { citados, } \\
\text { exercícios } \\
\text { para esta- } \\
\text { bilização } \\
\text { lombar ( } n=8 \text { ) }\end{array}$ & $\begin{array}{l}\text { O grupo } \\
\text { intervenção era } \\
\text { composto por } \\
8 \text { indivíduos. } \\
\text { Foram utiliza- } \\
\text { dos os jogos } \\
\text { Wakeboard, } \\
\text { Frisbe dog, Jet } \\
\text { Sky, Canoe. Os } \\
\text { participantes } \\
\text { escolhiam o } \\
\text { jogo de maior } \\
\text { interesse para } \\
\text { o atendimento } \\
\text { e faziam um } \\
\text { descanso de } \\
\text { dois minutos } \\
\text { a cada dez de } \\
\text { atendimento.O } \\
\text { atendimento } \\
\text { também foi } \\
\text { precedido pelo } \\
\text { uso dos recur- } \\
\text { sos eletrotér- } \\
\text { micos }\end{array}$ & $\begin{array}{l}\text { Três vezes } \\
\text { por semana, } \\
\text { durante oito } \\
\text { semanas, } \\
\text { com duração } \\
\text { de até } 45 \\
\text { minutos para } \\
\text { os recursos } \\
\text { eletrotérmicos } \\
\text { e aproxima- } \\
\text { damente } 30 \\
\text { minutos para } \\
\text { os exercícios de } \\
\text { estabilização } \\
\text { lombar ou o } \\
\text { uso do Ninten- } \\
\text { do Wii }\end{array}$ & $\begin{array}{l}\text { Escala Visual } \\
\text { Analógica } \\
\text { (EVA) para dor, } \\
\text { Força de eleva- } \\
\text { ção isomé- } \\
\text { trica para os } \\
\text { músculos das } \\
\text { costas, One-le- } \\
\text { gged Stand } \\
\text { Test, RAND-36 } \\
\text { Health Status } \\
\text { Inventory }\end{array}$ & $\begin{array}{l}\text { A dor diminuiu } \\
\text { significa- } \\
\text { tivamente } \\
\text { nos grupos } \\
\text { de exercício } \\
\text { e Wii. O } \\
\text { grupo Wii não } \\
\text { apresentou } \\
\text { melhora no } \\
\text { equilíbrio, po- } \\
\text { rém foi o único } \\
\text { com resultado } \\
\text { positivo sig- } \\
\text { nificativo na } \\
\text { avaliação de } \\
\text { aspectos emo- } \\
\text { cionais, como } \\
\text { bem estar e } \\
\text { problemas } \\
\text { emocionais }\end{array}$ \\
\hline
\end{tabular}


Tabela 1 - Sumário dos estudos que empregaram o Nintendo Wii para Reabilitação Traumato-ortopédica

(continuação)

\begin{tabular}{|c|c|c|c|c|c|c|c|c|}
\hline AUTOR/ANO & PAís & $\begin{array}{l}\text { TIPO DE } \\
\text { ESTUDO }\end{array}$ & AMOSTRA & $\begin{array}{c}\text { GRUPO } \\
\text { CONTROLE }\end{array}$ & INTERVENÇÃO & $\begin{array}{l}\text { DURAÇÃO DO } \\
\text { PROGRAMA }\end{array}$ & $\begin{array}{l}\text { INSTRUMENTOS } \\
\text { DE AVALIAÇÃO }\end{array}$ & CONCLUSÕES \\
\hline $\begin{array}{l}\text { Baltaci, } \\
\text { Harput, } \\
\text { Haksever, } \\
\text { Ulusoy, } \\
\text { Ozer (2012) }\end{array}$ & Turquia & $\begin{array}{l}\text { Randomiza- } \\
\text { do, clínico, } \\
\text { controlado, } \\
\text { duplo-cego, } \\
\text { prospectivo }\end{array}$ & $\begin{array}{l}30 \text { indivíduos } \\
\text { submetidos a } \\
\text { reconstrução } \\
\text { do ligamen- } \\
\text { to cruzado } \\
\text { anterior } \\
\text { do joelho } \\
\text { com uso de } \\
\text { tendão de } \\
\text { isquiotibiais }\end{array}$ & $\begin{array}{l}\text { Mobilização } \\
\text { precoce, } \\
\text { descarga } \\
\text { de peso de } \\
\text { acordo com } \\
\text { a tolerân- } \\
\text { cia, flexão } \\
\text { em cadeia } \\
\text { cinética } \\
\text { fechada, } \\
\text { fortaleci- } \\
\text { mento de } \\
\text { quadríceps, } \\
\text { bicicleta, } \\
\text { exercícios } \\
\text { de equi- } \\
\text { líbrio. Os } \\
\text { exercícios } \\
\text { foram pro- } \\
\text { gredindo de } \\
\text { acordo com } \\
\text { o tempo e } \\
\text { evolução } \\
\text { do paciente } \\
\text { (n=15) }\end{array}$ & \begin{tabular}{|l} 
Foram \\
utilizados os \\
jogos Bowling \\
(boliche), Skiing \\
(ski), Boxing \\
(boxe), Football \\
(futebol) e \\
Sports Pro \\
Series com o \\
uso da Balance \\
Board ( $n=15$ )
\end{tabular} & $\begin{array}{l}\text { Três vezes por } \\
\text { semana, com } \\
\text { duração de } 60 \\
\text { minutos por } \\
\text { sessão, durante } \\
12 \text { semanas }\end{array}$ & $\begin{array}{l}\text { Modified } \\
\text { star excur- } \\
\text { sion balance } \\
\text { test (SEBT), } \\
\text { sistema de } \\
\text { agachamento } \\
\text { functional com } \\
\text { monitorização } \\
\text { computadori- } \\
\text { zada, (teste de } \\
\text { coordenação, } \\
\text { propriocepção } \\
\text { e tempo-res- } \\
\text { posta), força } \\
\text { concêntrica de } \\
\text { joelho através } \\
\text { de dinamôme- } \\
\text { tro isocinético }\end{array}$ & $\begin{array}{l}\text { Ambos os } \\
\text { grupos foram } \\
\text { efetivos nas } \\
\text { variáveis ana- } \\
\text { lisadas, porém } \\
\text { não houve } \\
\text { diferença } \\
\text { significativa } \\
\text { na compara- } \\
\text { ção entre as } \\
\text { modalidades } \\
\text { terapêuticas }\end{array}$ \\
\hline $\begin{array}{l}\text { Hsu, } \\
\text { Thibodeau, } \\
\text { Wong, } \\
\text { Zukiwsky, } \\
\text { Cecile, } \\
\text { Walton } \\
\text { (2011) }\end{array}$ & Canadá & $\begin{array}{l}\text { Randomiza- } \\
\text { do, cruzado, } \\
\text { cego-sim- } \\
\text { ples }\end{array}$ & $\begin{array}{l}34 \text { indivíduos } \\
\text { residentes } \\
\text { em um centro } \\
\text { de longa } \\
\text { permanên- } \\
\text { cia com } \\
\text { disfunção na } \\
\text { extremidade } \\
\text { do membro } \\
\text { superior (dor, } \\
\text { fraqueza, } \\
\text { rigidez, entre } \\
\text { outros) }\end{array}$ & $\begin{array}{l}\text { Programa } \\
\text { de exercício } \\
\text { incluindo } \\
\text { fortaleci- } \\
\text { mento de } \\
\text { membros } \\
\text { superiores } \\
\text { e inferiores, } \\
\text { exercícios } \\
\text { de coor- } \\
\text { denação e } \\
\text { equilíbrio }\end{array}$ & $\begin{array}{l}\text { Participação } \\
\text { do programa } \\
\text { de exercícios, } \\
\text { adicionando } \\
\text { a este o jogo } \\
\text { Bowling (bo- } \\
\text { liche) no Wii , } \\
\text { jogando com o } \\
\text { braço mais afe- } \\
\text { tado segundo } \\
\text { auto-relato }\end{array}$ & $\begin{array}{l}\text { O programa } \\
\text { de exercícios } \\
\text { aconteceu de } \\
\text { duas a quatro } \\
\text { vezes por } \\
\text { semana. A uti- } \\
\text { lização do Wii } \\
\text { foi empregada } \\
\text { duas vezes } \\
\text { por semana } \\
\text { por cerca de } \\
20 \text { minutos/ } \\
\text { sessão. Após } \\
4 \text { semanas os } \\
\text { grupos eram } \\
\text { cruzadose se } \\
\text { seguiam mais } \\
4 \text { semanas de } \\
\text { atendimento }\end{array}$ & $\begin{array}{l}\text { Nursing Home } \\
\text { Physical Test } \\
\text { (NHPPT), } \\
\text { versão mo- } \\
\text { dificada do } \\
\text { Physical Acti- } \\
\text { vity Enjoyment } \\
\text { Scale (PACES), } \\
\text { Numeric Rating } \\
\text { Scale (NRS), } \\
\text { amplitude de } \\
\text { movimento } \\
\text { ativa, Global } \\
\text { Perceived Ra- } \\
\text { ting Changes } \\
\text { (GPRC) }\end{array}$ & $\begin{array}{l}\text { Os participan- } \\
\text { tes apresenta- } \\
\text { ram melhora } \\
\text { na maioria } \\
\text { das variáveis } \\
\text { estudadas. O } \\
\text { grupo Wii não } \\
\text { foi eficaz no } \\
\text { gerenciamento } \\
\text { da dor, porém } \\
\text { apresentou } \\
\text { melhora } \\
\text { significativa no } \\
\text { amplitude de } \\
\text { movimento e é } \\
\text { um comple- } \\
\text { mento útil } \\
\text { aos exercícios } \\
\text { convencionais }\end{array}$ \\
\hline
\end{tabular}

\section{DISCUSSÃO}

A realidade virtual é um recente coajduvante no tratamento dos mais diversos tipos de distúrbios cinético-funcionais. $O$ estudo presente teve como objetivo avaliar a existência de pesquisas que descrevam a experiência da wiiterapia na reabilitação de disfunções traumato-ortopédicas.
O emprego do console se deu através de diferentes jogos e em uma gama, mesmo que limitada, de casos, com destaque para reabilitação da articulação do joelho. O tratamento baseado no Nintendo Wii ${ }^{\circledR}$ como componente central ou adjuvante na modalidade convencional, se mostrou na maior parte dos estudos tão eficaz quanto o tratamento baseado na fisioterapia tradicional, com exercícios de fortalecimento, equilíbrio e alongamento, apresentando como diferencial o aspecto de motivação 
e diversão, raramente frequentes em um programa pautado puramente no exercício, embora este mesmo sistema de pontuação seja capaz de desestimular o praticante após sucessivos resultados negativos.

Em estudo randomizado cruzado, realizado com idosos residentes em instituições de longa permanência (ILP) com disfunção no membro superior (quadro álgico ou rigidez), os pesquisadores compararam o programa de exercício convencional com um programa que associava os exercícios convencionais à prática do Wii Bowling $®$ (boliche). A percepção de dor, limitações do movimento e nível de diversão com as ações realizadas no jogo foram avaliadas. Os resultados sugeriram que a adição do Nintendo Wiiß a programas terapêuticos nesse grupo populacional é extremamente útil, destacando a wiiterapia como uma abordagem motivadora ao tratamento. ${ }^{(18)}$ Grande parte dos estudos que abordaram a relação entre o Nintendo Wii ${ }^{\circ}$ e suas repercussões terapêuticas para membros superiores envolveram indivíduos com comprometimento funcional pós Acidente Vascular Encefálico, ${ }^{(19,20)} \mathrm{com}$ resultados positivos. $O$ incentivo à mobilização ativa de membros parcialmente dependentes, preconizado por recursos tecnológicos como o Nintendo $\mathrm{Wii} @$, pode ter sido fator preponderante, com possível interferência na plasticidade neuronal em atividades até então limitadas funcionalmente.

Utilizando a realidade virtual por meio do Nintendo Wii $\circledast$ com um programa pautado em jogos de fortalecimento, condicionamento e ioga, Sims et al. ${ }^{(21)}$ buscaram determinar os efeitos da terapêutica na funcionalidade e no controle postural de pacientes com disfunção de membros inferiores, comparando com o tratamento convencional e com um grupo controle ao qual foi recomendado que continuassem com suas atividades cotidianas normais. A utilização do Nintendo $\mathrm{Wii} \circledast$ se mostrou útil na otimização do controle postural nos indivíduos analisados. O equilíbrio postural e a força muscular de membros inferiores também foram avaliados em idosos residentes na comuni- dade submetidos a um protocolo de intervenção com Nintendo Wii ${ }^{\circledR}$ com 10 semanas de duração, apresentando resultados superiores aos encontrados no grupo controle. ${ }^{(22)}$ Importante salientar que respostas posturais inadequadas apresentam forte correlação a quedas, ${ }^{(23)}$ e que estas podem vir em detrimento à redução de força muscular em membros inferiores.

Em revisão conduzida por Laufer, Dar, Kodesh acerca do uso do Nintendo $\mathrm{Wii} \circledast$ para o incremento do equilíbrio em idosos funcionalmente independentes, concluiu-se que o console estudado pode ser utilizado como uma ferramenta alternativa aos programas de treinamento convencionais, destacando-se pelo seu potencial de entretenimento associado à eficácia da terapêutica. ${ }^{(24)}$

O estudo realizado no Brasil(25) buscou investigar a aplicação do Nintendo Wii $\circledast$ em mulheres com dor e disfunção decorrentes de osteoartrite do joelho. Foram selecionados jogos de ioga e equilíbrio com a utilização da balance board $($, dispositivo que permite o reconhecimento da mudança no centro de gravidade em função do movimento corporal. Ao compararem o programa de realidade virtual com exercícios convencionais, os pesquisadores verificaram que a wiiterapia foi mais eficaz nas variáveis rigidez e equilíbrio e menos eficaz no que diz respeito à escores de dor e incapacidade, quando comparado aos exercícios convencionais. Nesse sentido, cabe destacar um estudo de revisão sistemática, ${ }^{(26)}$ que atribuiu à força muscular de coxa, à melhora do movimento de extensão de joelhos e à melhora na propriocepção como os possíveis mediadores na associação positiva entre o exercício físico e os sintomas da osteoartrite em joelhos, sendo que a duração e a intensidade desse exercício devem ser aplicados com parâmetros adequados.

Fung et al. (2) avaliaram a utilização da realidade virtual na reabilitação de indivíduos após artroplastia total do joelho. Um programa de quinze minutos de wiiterapia com a adoção de jogos para equilíbrio postural com deslocamentos lateral e multidirecional foi utilizado pelo grupo inter- 
venção, enquanto o grupo controle foi submetido a um programa de fisioterapia convencional de mesma duração. Não houveram diferenças significativas entre os dois grupos. O programa de realidade virtual se mostrou eficaz na reabilitação destes pacientes, com potencial para atuar como ferramenta de continuidade de tratamento no domićlio. Uma das maiores aplicações da realidade virtual no campo da reabilitação do equilíbrio postural se refere ao seu uso no campo da neurologia, especialmente com pacientes portadores de Doença de Parkinson. Uma revisão buscou investigar qual a função que os chamados exergames geram no equilíbrio e em outros fatores, destacando aspectos positivos desta ferramenta, como sua efetividade na otimização do equilíbrio e na redução dos sintomas motores da doença, porém também é apontado que a complexidade e velocidade dos jogos podem se mostrar como um ponto negativo para 0 participante da reabilitação. ${ }^{(8)}$

Ainda com relação à articulação do joelho, em um estudo prospectivo randomizado, controlado e duplo cego, os autores investigaram um programa de reabilitação com a realidade virtual em indivíduos submetidos à reconstrução do ligamento cruzado anterior. Os exercícios no Nintendo Wiiß foram o boliche, sky, boxe e futebol, sendo escolhidos devido ao potencial na reabilitação desta modalidade de lesão. O grupo controle foi submetido a um programa de exercícios em cadeia cinética fechada, na fase inicial, até corrida, ao final do tratamento. Os dois grupos apresentaram resultados positivos, porém os achados quanto à superioridade de uma dada abordagem sobre a outra na restauração do controle motor e funcionalidade foi inconclusiva. (3)

Kim et al. ${ }^{(27)}$ investigaram um programa de reabilitação baseado em realidade virtual com o emprego do Wii Fit Yoga® em mulheres de meia idade com lombalgia comparado a um programa de exercícios para estabilização central do tronco, no sentido de minimizar o quadro álgico e a disfunção gerada por esta condição. Ambos os grupos apresentaram melhora na avaliação pré e pós intervenção. Importante salientar que, mesmo com pou- cos estudos abordando os mecanismos geradores das alterações no controle postural, é possível que ocorra uma correlação entre a postura alterada e as modificações sensório-motoras, pois a dor é um sintoma relevante para o surgimento de alterações mecânicas, frequentes em indivíduos com lombalgias, e que podem ser melhorados diante de protocolos de intervenção realizados pelo Nintendo Wiiß.

No âmbito laboral, Park, Lee e Ko(4) investigaram a utilização de um programa de wiiterapia no tratamento de lombalgia em trabalhadores industriais. Aos participantes, foi dada a oportunidade de es-

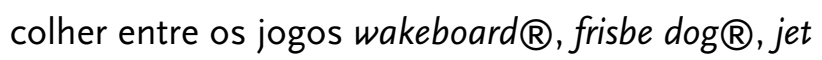
$s k y \circledast$ e canoe $囚$. O grupo controle foi submetido a um programa de exercício com fortalecimento de musculatura estabilizadora do tronco e ambos os grupos foram precedidos pela utilização de recursos eletrotermoterápicos para analgesia. O grupo que utilizou o Nintendo Wii $囚$ foi o único que apresentou resultados significativamente positivos nos componentes emocionais do questionário de qualidade de vida. Pressupõe-se que a realidade virtual encoraja os participantes a se divertirem enquanto realizam o exercício, apresentando portanto ganhos também no aspecto emocional do tratamento.

LeBlanc e colaboradores investigaram os efeitos dos video games ativos que promovem um maior empenho energético de seus participantes na área de saúde das crianças e adolescentes. Os resultados indicaram que video games ativos, como o Nintendo Wii $\circledast$, são capazes de aumentar a intensidade da atividade física ao patamar de atividade leve para moderada, mas sem dados claros sobre como esses jogos influenciam no comportamento sedentário dos indivíduos estudados. ${ }^{(28)}$

Uma outra aplicação da realidade virtual no campo da saúde é no combate à obesidade. Em estudo conduzido por Lu, Kharrazi, Gharghabi, Thompson, buscou-se investigar quais as características dos jogos são capazes de alcançar os objetivos voltados à perda de peso. Apesar de resultados positivos, ressaltou-se a carência que esta modalidade 
de jogos apresenta no aspecto técnico "enredo", sendo este um ponto que pode dificultar a adesão do paciente à modalidade de terapia pela ausência de história. ${ }^{(12)}$

Entretanto, cabe aqui a ressalva, principalmente quando se trata da área traumato-ortopédica, de que o emprego da wiiterapia também pode ser responsável por efeitos deletérios à saúde humana, perpassando desde lesões ligamentares, tendinites, risco de queda e consequente trauma durante a utilização da plataforma balance board dentre uma outra série de lesões traumáticas causadas pelo impacto do controle com um jogador próximo tanto no modo cooperativo, quanto no modo versus um outro participante. ${ }^{(29)}$

\section{CONSIDERAÇÕES FINAIS}

O surgimento e fenômeno de popularização dos exergames fez com que os profissionais do segmento da reabilitação buscassem compreender se esta ferramenta poderia apresentar viabilidade, eficiência e aumento da interação do paciente com o seu próprio processo de recuperação. O Nintendo Wii é um console fortemente difundido no mundo, sendo por isso alvo de vários estudos que buscaram incluí-lo como protagonista ou colaborador nos programas de reabilitação.

O presente estudo mostrou que, embora com aplicações até agora limitadas à poucas condições traumato-ortopédicas, a wiiterapia, como é conhecida a utilização desta plataforma para a recuperação funcional de indivíduos com algum problema de saúde, se mostrou eficaz, atuando tanto em condições de acometimento de membros superior, inferior ou coluna.

A pesquisa apresentou, como limitação central, a dificuldade em se encontrar estudos que buscassem a relação entre a wiiterapia e a reabilitação traumato-ortopédica. Diante disto, recomenda-se que mais pesquisas sejam feitas na área para uma real noção da extensão da utilização que - Nintendo Wii pode alcançar nessa modalidade terapêutica.

\section{REFERÊNCIAS}

1. Tavares CN, Carbonero FC, Finamore PS, Kós RS. Uso do Nintendo ${ }^{\circledR}$ Wii para Reabilitação de Crianças com Paralisia Cerebral: Estudo de caso. Rev. Neurocienc. 2013;21 (2):286-293.

2. Fung V, Ho A, Shaffer J, Chung E, Gomez M. Use of Nintendo Wii Fit ${ }^{T M}$ in the rehabilitation of outpatients following total knee replacement: a preliminary randomised controlled trial. Physiotherapy. 2012;98(3):183-8.

3. Baltaci G, Harput G, Haksever B, Ulusoy B, Ozer H. Comparison between Nintendo Wii Fit and conventional rehabilitation on functional performance outcomes after hamstring anterior cruciate ligament reconstruction: prospective, randomized, controlled, doubleblind clinical trial. Knee surg. sports traumatol. arthrosc. 2013;21(4):880-7.

4. Park J.-H., Lee S.-H., Ko D.-S. (2O13). The Effects of the Nintendo Wii Exercise Program on Chronic Work-related Low Back Pain in Industrial Workers. J. phys. ther. sci. 2013;25(8), 985-988.

5. Jansen-Kosterink SM, Huis In 't Veld RM, Schönaver C, Kaufmann H, Hermens HJ, Vollenbroek-Hutten MM. A Serious Exergame for Patients Suffering from Chronic Musculoskeletal Back and Neck Pain: A Pilot Study. Games Health J. 2013;2(5):299-307.

6. Souza RA, Cruz LG, Carvalho Priscila Silva de, Silva FF, Carvalho WRG. Respostas cardiovasculares agudas em ambiente virtualmente simulado pelo Nintendo Wii. Rev. bras. cineantropom. desempenho hum. 2013;15(1):60-70.

7. Gonçalves GB, Leite MAA, Orsini M, Pereira JS. Effects of Using the Nintendo Wii Fit Plus Platform in the Sensorimotor Training of Gait Disorders in Parkinson's Disease. Neurol. int. 2014;6(1):5048.

8. Barry G, Galna B, Rochester L. The role of exergaming in Parkinson's disease rehabilitation: a systematic review of the evidence. J Neuroeng Rehabil. 2014;11:33.

9. Cacau LA, Oliveira GU, Maynard LG, Araújo Filho AA, Silva WM Jr, Cerqueria Neto ML, Antoniolli AR, Santana-Filho VJ. The use 
of the virtual reality as intervention tool in the postoperative of cardiac surgery. Rev. bras. cir. cardiovasc. 2013;28(2):281-9.

10. Bosch PR, Poloni J, Thornton A, Lynskey JV. The Heart Rate Response to Nintendo Wii Boxing in Young Adults. Cardiopulm. phys. ther. j. 2Ol2;23(2):13-29.

11. Staiano AE, Abraham AA, Calvert SL. The Wii Club: Gaming for Weight Loss in Overweight and Obese Youth. Games Health J. 2012;1(5):377-380.

12. Lu AS, Kharrazi H, Gharghabi F, Thompson D. A Systematic Review of Health Videogames on Childhood Obesity Prevention and Intervention. Games Health J. 2013;2(3):131-141.

13. Harrison M. No Wii cause for concern. Emerg. med. j. 2009;26:150.

14. Bonis J. Acute Wiiitis. N. Engl. j. med. 2007;356:2431-2.

15. Rubin D. Triad of spinal pain, spinal joint dysfunction, and extremity pain in 4 pediatric cases of "Wii-itis": a 21st century pediatric condition. J. chiropr. med. 2010;9:84-9.

16. Choi JH, Han EY, Kim BR, Kim SM, Im SH, Lee $\mathrm{SY}, \mathrm{Hyun} \mathrm{CW}$. Effectiveness of commercial gaming-based virtual reality movement therapy on functional recovery of upper extremity in subacute stroke patients. Ann Rehabil Med. 2014;38(4):485-93.

17. Gonçalves GB, Leite MA, Orsini M, Pereira $J S$. Effects of using the nintendo wii fit plus platform in the sensorimotor training of gait disorders in Parkinson's disease. Int. j. neurol. 2014;6(1):5048.

18. Hsu JK, Thibodeau R, Wong SJ, Zukiwsky D, Cecile S, Walton DM. A "Wii" bit of fun: the effects of adding Nintendo Wii( $\left.{ }^{\circledR}\right)$ Bowling to a standard exercise regimen for residents of long-term care with upper extremity dysfunction. Physiother. theory pract. 2011;27(3):185-93.

19. Peters DM, McPherson AK, Fletcher B, McClenaghan BA, Fritz SL. Couting repetitions: an observational study of video game play in people with chronic poststroke hemiparesis. J. neuro. phys. ther. 2013;37(3):105-11.
2O. Saposnik G, Teasell R, Marndani M, Hall J, Mcllroy W, Cheung D, Thorpe KE, Cohen LG, Bayley M, Stroke Outcome Research Canada (SORCan) Working Group. Stroke. 2010;41(7):1477-84.

21. Sims J, Cosby N, Saliba EN, Hertel J, Saliba SA. Exergaming and static postural control in individuals with a history of lower limb injury. J. athl. train. 2013;48(3):314-25.

22. Jorgensen MG, Laessoe U, Hendriksen C, Nielsen OB, Aagaard P. Efficacy of Nintendo Wii training on mechanical leg muscle function and postural balance in community-dwelling older adults: a randomized controlled trial. J. gerontol. 2013;68(7):845-52.

23. Jacobs JV. Why we need to better understand the cortical neurophysiology of impaired postural responses with age, disease, or injury. Front. integr. neurosci. 2014;8:69.

24. Laufer Y, Dar G, Kodesh E. Does a Wii-base exercise program enchance balance control of independently funcioning older adults? A systematic review. Clin. interv. aging. 2014:9; 1803-1813.

25. 25. Wibelinger LM, Batista JS, Vidmar MF, Kayser B, Pasqualotti A, Schneider RH. Efeitos da fisioterapia convencional e da wiiterapia na dor e capacidade funcional de mulheres idosas com osteoartrite de joelho. Rev. dor. 2013;14(3):196-199.

26. Runhaar J, Luijsterburg P, Dekker J, BiermaZeinstra SM. Identifying potential working mechanisms behind the positive effects of exercise therapy on pain and function in osteoarthritis: a systematic review. Osteoarthr. cartil. 2015 Apr 9.

27. Kim S.-S., Min W.-K., Kim J.-H., Lee, B.-H. (2O14) The Effects of VR-based Wii Fit Yoga on Physical Function in Middle-aged Female LBP Patients. J. phys. ther. sci. 2014;26(4),549-552.

28. LeBlanc AG, Chaput J-P, McFarlane A, Colley RC, Thivel D, et al. Active Video Games and Health Indicators in Children and Youth: A SystematicReview. PLoS ONE. 2013; 8(6).

29. Fysh T, Thompson JF. A Wii Problem. J R Soc Med 2009;102:501-502. 